\title{
Communication \\ Gravitational Radiation as the Bremsstrahlung of Superheavy Particles in the Early Universe
}

\author{
Andrey A. Grib ${ }^{1,2, *}$ and Yuri V. Pavlov ${ }^{3,4}$ \\ 1 Theoretical Physics and Astronomy Department, The Herzen University, 48 Moika, \\ 191186 St. Petersburg, Russia \\ 2 A. Friedmann Laboratory for Theoretical Physics, 191186 St. Petersburg, Russia \\ 3 Institute of Problems in Mechanical Engineering of Russian Academy of Sciences, 61 Bolshoy, V.O., \\ 199178 St. Petersburg, Russia; yuri.pavlov@mail.ru \\ 4 N.I. Lobachevsky Institute of Mathematics and Mechanics, Kazan Federal University, 420008 Kazan, Russia \\ * Correspondence: andrei_grib@mail.ru
}

Received: 15 September 2020; Accepted: 15 October 2020; Published: 20 October 2020

check for updates

\begin{abstract}
The number of superheavy particles with the mass of the Grand Unification scale with trans-Planckian energy created at the epoch of superheavy particle creation from the vacuum by the gravitation of the expanding Universe is calculated. In later collisions of these particles, gravitational radiation is radiated playing the role of bremsstrahlung for gravity. The effective background radiation of the Universe is evaluated.
\end{abstract}

Keywords: particle creation; early Universe; Grand Unification

\section{Introduction}

In the paper published by one of the present authors (A.A.G.) together with S.G. Mamayev [1], finite results for particle creation in the early expanding Friedmann Universe were obtained. The important result was the calculation of the finite density and finite total number of particles created in the Lagrange volume. Our results were obtained by using Fock quantization with the vacuum defined as the ground state of the instantaneous Hamiltonian. These results have a simple intuitive physical interpretation due to the work done on the virtual pair on the Compton length of the particle by the tidal forces due to the gravity of the expanding Universe. The number of particles depends on the mass of the particle and leads to observable numbers of visible and dark matter particles if this mass identified with the mass of the dark matter particles is equal to the number close to the Great Unification scale [2]. A possible explanation of the origin of ultra high energy cosmic rays due to the decay of such particles was given in [3-6].

Therefore, the main idea is that superheavy particles were created by gravitation, then some part of them decayed to visible particles at high energies; however, after the energy became smaller, the decays were frozen, and surviving superheavy particles formed the observed dark matter [3]. However, the mathematical calculation of particle creation makes possible the calculation of the distribution function depending on the momenta, i.e., density not in coordinate, but in momentum space. Surely, the finiteness of the particle density in coordinate space means that this function is going to zero at very high values of the momentum. This means that the larger the momentum, the smaller will be the number of created particles.

In this paper, we shall answer the question about the number of superheavy particles with Grand Unification mass, but with the energy close to the Planckian mass. How many trans-Planckian particles are created? Surely, their number is much smaller than the general number of created particles, but how much? For the case of inflationary models, a similar question was asked for same particles called 
wimpzillas [7]. Why are this question and the answer to it important? This is because in our recent paper [8], it was found that at trans-Planckian energies of colliding particles, gravitation wave radiation is produced. This radiation is much stronger than the comparable electromagnetic radiation if it could exist. It plays the role of the bremsstrahlung for superheavy particle and can lead to the formation of structures for them as is the case for electromagnetic radiation at small energies when galaxies, stars, etc., can be formed. In the paper [9], it was also shown that collision of particles with trans-Planckian energies can lead to the formation of mini black holes. The problem of the formation of primary galactic nuclei during the phase transition in the early Universe was considered in [10].

In the book [11], it was mentioned that in a Friedmann expanding Universe, the number of created particles is proportional to the number of causally disconnected parts of the Universe at the Compton time of the existence of the Universe. However, later, these disconnected parts are united, and collisions of particles and black holes occur. This makes possible the speculation of the possible arising of the primordial black holes leading to the active nuclei of Galaxies by this mechanism.

\section{Creation of Superheavy Particles with Planckian Energy in the Early Universe}

Consider the creation of scalar and spinor particles in the early homogeneous isotropic Friedmann Universe with metric:

$$
d s^{2}=g_{i k} d x^{i} d x^{k}=a^{2}(\eta)\left(d \eta^{2}-d l^{2}\right)
$$

where $d l^{2}$ is the metric of a three-dimensional space of constant curvature $K=0, \pm 1$.

In the theory of quantum effects in expanding curved space-time, one usually takes the following equation (in the system of units in which the Planck constant and light velocity are equal to one: $\hbar=c=1$ ) for a scalar field of mass $m$ :

$$
\left(\nabla^{i} \nabla_{i}+\xi R+m^{2}\right) \varphi(x)=0
$$

corresponding to the Lagrangian:

$$
L(x)=\sqrt{|g|}\left[g^{i k} \partial_{i} \varphi^{*} \partial_{k} \varphi-\left(m^{2}+\xi R\right) \varphi^{*} \varphi\right],
$$

where $g=\operatorname{det}\left(g_{i k}\right)$ and $R$ is the curvature scalar [12].

For $\xi=\xi_{c} \equiv 1 / 6$, the scalar field is called conformal coupled. Then, Equation (2) is conformally invariant in the massless case [2]. However, the nonconformal case is also important because (1) "gravitons" [13] and (2) longitudinal components of vector bosons [2] are nonconformal. Minimal coupling $\xi=0$ is popular in inflation theories [14].

We can find a complete set of solutions for Equation (2) in the following form:

$$
\varphi(x)=\frac{g_{\lambda}(\eta)}{a(\eta)} \Phi_{J}(\mathbf{x}),
$$

where:

$$
\begin{gathered}
g_{\lambda}^{\prime \prime}(\eta)+\Omega^{2}(\eta) g_{\lambda}(\eta)=0, \\
\Omega^{2}(\eta)=\left(m^{2}+\left(\xi-\xi_{c}\right) R\right) a^{2}+\lambda^{2}, \\
\Delta \Phi_{J}(\mathbf{x})=-\left(\lambda^{2}-K\right) \Phi_{J}(\mathbf{x}) ;
\end{gathered}
$$

the prime denotes a derivative with respect to conformal time $\eta$, and index $J$ numbers the eigenfunctions of Laplace-Beltrami operator $\Delta$ in space $\mathbf{x}$ with metric $d l^{2}$.

According to the Hamiltonian diagonalization method [2], the functions $g_{\lambda}(\eta)$ satisfy initial conditions:

$$
g_{\lambda}^{\prime}\left(\eta_{0}\right)=i \Omega\left(\eta_{0}\right) g_{\lambda}\left(\eta_{0}\right), \quad\left|g_{\lambda}\left(\eta_{0}\right)\right|=\frac{1}{\sqrt{\Omega\left(\eta_{0}\right)}} .
$$


The number of pairs of scalar particles created up to the moment $t$ in Lagrangian volume $a^{3}(t)$ of a homogeneous isotropic Universe with flat space sections $(K=0)$ is equal [2]:

$$
N(\eta)=\frac{1}{2 \pi^{2}} \int_{0}^{\infty} s_{\lambda}(\eta) \lambda^{2} d \lambda,
$$

where:

$$
s_{\lambda}(\eta)=\frac{\left|g_{\lambda}^{\prime}(\eta)-i \Omega g_{\lambda}(\eta)\right|^{2}}{4 \Omega} .
$$

Function $s_{\lambda}(\eta)$ defines the distribution in dimensionless momentum $\lambda$ particles created up to moment $\eta$. The "physical" momentum is $\lambda / a$. For ultrarelativistic particles, $\lambda \gg m a$. To find the number of created ultrarelativistic particles, one must find the asymptote of the function $s_{\lambda}(\eta)$ if $\lambda \rightarrow \infty$.

Using (5) and (8), one can see that function $s_{\lambda}(t)$ satisfies the integral Volterra equation:

$$
s_{\lambda}(\eta)=\frac{1}{2} \int_{\eta_{0}}^{\eta} d \eta_{1} w\left(\eta_{1}\right) \int_{\eta_{0}}^{\eta_{1}} d \eta_{2} w\left(\eta_{2}\right)\left(1+2 s_{\lambda}(\eta)\right) \cos \left[2 \Theta\left(\eta_{2}, \eta_{1}\right)\right],
$$

where:

$$
w(\eta)=\frac{\Omega^{\prime}(\eta)}{\Omega}, \quad \Theta\left(\eta_{2}, \eta_{1}\right)=\int_{\eta_{1}}^{\eta_{2}} \Omega(\eta) d \eta
$$

To find the asymptote $s_{\lambda}(\eta)$ if $\lambda \rightarrow \infty$, one can confine one's self to the first iteration of the integral Equation (11) and take into account that $\Theta\left(\eta_{2}, \eta_{1}\right) \rightarrow \lambda\left(\eta_{1}-\eta_{2}\right)$ if $\lambda \rightarrow \infty$. Then, (11) has the form:

$$
s_{\lambda}(\eta)=\frac{1}{4}\left|\int_{\eta_{0}}^{\eta} w\left(\eta_{1}\right) \exp \left(2 i \lambda \eta_{1}\right) d \eta_{1}\right|^{2} .
$$

Integrating by parts the integral (13), one obtains in $O\left(\lambda^{-2}\right)$ :

$$
\left.\int_{\eta_{0}}^{\eta} w\left(\eta_{1}\right) \exp \left(2 i \lambda \eta_{1}\right) d \eta_{1} \approx \frac{w(\eta)}{2 i \lambda} e^{2 i \lambda \eta}\right|_{\eta_{0}} ^{\eta} .
$$

Therefore,

$$
s_{\lambda}(\eta) \approx \frac{1}{16 \lambda^{2}} \mid w^{2}(\eta)+w^{2}\left(\eta_{0}\right)-2 w(\eta) w\left(\eta_{0}\right) \cos \left(2 \lambda\left(\eta-\eta_{0}\right) \mid .\right.
$$

For $\lambda \rightarrow \infty$, one has that $w \sim \lambda^{-2}$, so $s_{\lambda} \sim \lambda^{-6}$, and the integral in (9) is convergent. Therefore, in the method of Hamiltonian diagonalization, the number of scalar particles created by gravitation is finite for conformal as for nonconformal coupling with curvature.

Evaluate the number of created particles with the energy $E \geq E_{b}$, where $E_{b} \gg m c^{2}$. The cyclic frequency $\Omega$ for the scalar field with conformal coupling with curvature is:

$$
\Omega(\eta)=\omega(\eta) \equiv \sqrt{m^{2} a^{2}(\eta)+\lambda^{2}} .
$$

In this case:

$$
w(\eta)=\frac{a^{\prime} / a}{1+\left(\frac{\lambda}{m a}\right)^{2}} .
$$

For large $\lambda$ :

$$
\lambda \gg m a \Rightarrow w \approx \frac{m^{2} a^{\prime} a}{\lambda^{2}} .
$$


Let us consider the situation when $a^{\prime} a$ is increasing in time and $a^{\prime}\left(\eta_{0}\right) a\left(\eta_{0}\right) \ll a^{\prime}(\eta) a(\eta)$. Then:

$$
s_{\lambda}(\eta) \approx \frac{w^{2}(\eta)}{16 \lambda^{2}}=\frac{m^{4} a^{2} a^{\prime 2}}{16 \lambda^{6}}, \lambda \rightarrow \infty .
$$

Find what limitations on the background matter of the Universe arise due to this condition. Einstein's equations:

$$
R_{i k}-\frac{1}{2} g_{i k} R=-8 \pi G T_{i k},
$$

for homogeneous isotropic space in metric (1) are:

$$
\begin{gathered}
\frac{1}{a^{2}}\left(\left(\frac{a^{\prime}}{a}\right)^{2}+K\right)=\frac{8 \pi G \varepsilon}{3}, \\
-\frac{1}{a^{2}}\left[\left(\frac{a^{\prime}}{a}\right)^{\prime}+\frac{1}{2}\left(\left(\frac{a^{\prime}}{a}\right)^{2}+K\right)\right]=4 \pi G p,
\end{gathered}
$$

where $\varepsilon$ and $p$ are the energy density and pressure for background matter. From (21) and (22), one has:

$$
\left(a^{\prime} a\right)^{\prime}=\frac{a^{2}}{2}\left[3\left(1-\frac{p}{\varepsilon}\right)\left(\frac{a^{\prime}}{a}\right)^{2}-\left(1+3 \frac{p}{\varepsilon}\right) K\right] .
$$

For realistic models of the Universe in the epoch when the effects of particle creation are important (the Compton time of the particle [2]), one can neglect the space curvature and consider $\left|a^{\prime} / a\right| \gg 1$. Therefore,

$$
\left(a^{\prime} a\right)^{\prime} \approx \frac{3}{2}\left(1-\frac{p}{\varepsilon}\right) a^{\prime 2}
$$

and $a^{\prime} a$ is increasing if $p<\varepsilon$. The important cases of dust $p=0, a=a_{1} \eta=a_{0} t^{2 / 3}$, and radiation dominated Universes $p=\varepsilon / 3, a=a_{1} \eta=a_{0} \sqrt{t}$ are in this family. Here, $t$ is the coordinate time $d t=a d \eta$. The limiting case with scale factor $a=a_{1} \sqrt{\eta}=a_{0} t^{1 / 3}$ corresponds to the most rigid state equation $p=\varepsilon$.

Using (19), one obtains that the number of particle pairs with momenta larger than some $\lambda_{b}$, created in volume $a^{3}(t)$ of a homogeneous isotropic Universe is equal to:

$$
N_{b}(\eta) \approx \frac{1}{32 \pi^{2}} \int_{\lambda_{b}}^{\infty} \frac{m^{4} a^{2}(\eta) a^{\prime 2}(\eta) d \lambda}{\lambda^{4}}=\frac{m^{4} a^{2}(\eta) a^{\prime 2}(\eta)}{96 \pi^{2} \lambda_{b}^{3}} .
$$

The usual unit system is made by:

$$
m \rightarrow \frac{m c}{\hbar}, \quad a^{\prime} \rightarrow \frac{\dot{a} a}{c},
$$

where the dot above the symbols is the derivative with respect to time $t$. For ultrarelativistic particles with $E_{b}=\lambda_{b} \hbar c / a$ and so (25) in usual units:

$$
N_{b}(t) \approx \frac{m^{4} c^{5} a \dot{a}^{2}}{96 \pi^{2} \hbar E_{b}^{3}} .
$$

For the scale factor $a \sim t^{\alpha}$, one obtains:

$$
N_{b}(t) \approx \frac{\alpha^{2} m^{4} c^{5} a^{3}}{96 \pi^{2} \hbar E_{b}^{3} t^{2}} .
$$


For Planckian energy $E_{b}=E_{P l} \equiv \sqrt{\hbar c^{5} / G}$, where $G$ is the gravitational constant and the Compton time $t=t_{C} \equiv \hbar /\left(m c^{2}\right)$, one obtains:

$$
E_{b}=E_{P l} \Rightarrow N_{b}\left(t_{C}\right) \approx \frac{\alpha^{2}}{96 \pi^{2}}\left(\frac{l_{P l}}{l_{C}}\right)^{3}\left(\frac{a\left(t_{C}\right)}{l_{C}}\right)^{3},
$$

where $l_{C}=\hbar /(m c)$ is the Compton length of the particle and $l_{P l}=\sqrt{\hbar G / c^{3}}$ is the Planckian length. For the radiation dominated case $(\alpha=1 / 2)$, one obtains:

$$
N_{b}\left(t_{C}\right) \approx \frac{1}{384 \pi^{2}}\left(\frac{l_{P l}}{l_{C}}\right)^{3 / 2}\left(\frac{a\left(t_{P l}\right)}{l_{C}}\right)^{3} .
$$

Note that this result is valid not only for conformal, but also for nonconformal scalar particles because for the radiation dominated case $R=0$, and Formula (16) for the frequency (6) is correct for any value $\xi$ of the parameter of the connection of the scalar field with curvature.

For an observable Friedmann radiation dominated Universe, one obtains $a\left(t_{P l}\right) \approx 10^{-5} \mathrm{~m}$ so that for the scale of Grand Unification $m=10^{15} \mathrm{GeV}$, one has $10^{67}$ particles with the energy of the Planckian order. The general number of all superheavy particles created in the early Universe is close to the Eddington number $10^{80}$ [2]. Therefore, the number of trans-Planckian scalar particles is relatively small.

Now, consider the case of the creation of fermion particles. In this case, the number of created pairs is [2]:

$$
N^{(1 / 2)}(\eta)=\frac{1}{\pi^{2}} \int_{0}^{\infty} s_{\lambda}^{(1 / 2)}(\eta) \lambda^{2} d \lambda
$$

but the expression for $s_{\lambda}^{(1 / 2)}$ does not coincide with Formula (10). However, the first iteration in the integral equation for function $s_{\lambda}(\eta)$ coincides with Formula (13) if $w$ for the spinor field is:

$$
w^{(1 / 2)}=\frac{m a^{\prime} \lambda}{\omega^{2}}
$$

For large values $\lambda$ :

$$
\lambda \gg m a \Rightarrow w^{(1 / 2)} \approx \frac{m a^{\prime}}{\lambda}, \quad s_{\lambda}^{(1 / 2)} \approx \frac{m^{2} a^{\prime 2}}{16 \lambda^{4}} .
$$

The number of pairs of spinor particles with momenta larger than some $\lambda_{b}$, created in volume $a^{3}(t)$ of a homogeneous isotropic universe is equal to:

$$
N_{b}^{(1 / 2)}(\eta) \approx \frac{1}{16 \pi^{2}} \int_{\lambda_{b}}^{\infty} \frac{m^{2} a^{\prime 2}(\eta) d \lambda}{\lambda^{2}}=\frac{m^{2} a^{\prime 2}(\eta)}{16 \pi^{2} \lambda_{b}}
$$

in usual units:

$$
N_{b}^{(1 / 2)}(t) \approx \frac{m^{2} c a \dot{a}^{2}}{16 \pi^{2} \hbar E_{b}} .
$$

For the scale factor $a \sim t^{\alpha}$, one obtains that the number of created fermion pairs is:

$$
N_{b}^{(1 / 2)}(t) \approx \frac{\alpha^{2} m^{2} c a^{3}}{16 \pi^{2} \hbar E_{b} t^{2}} .
$$


For Planckian energy $E_{b}=E_{P l}$ and the Compton time, one obtains:

$$
E_{b}=E_{P l} \Rightarrow N_{b}^{(1 / 2)}\left(t_{C}\right) \approx \frac{\alpha^{2}}{16 \pi^{2}} \frac{l_{P l} a^{3}\left(t_{C}\right)}{l_{C}^{4}},
$$

in particular, for the radiation dominated case $(\alpha=1 / 2)$, one obtains:

$$
N_{b}^{(1 / 2)}\left(t_{C}\right) \approx \frac{1}{64 \pi^{2}} \frac{a^{3}\left(t_{P l}\right)}{l_{P l}^{1 / 2} l_{C}^{5 / 2}}
$$

For an observable Friedmann radiation dominated Universe, one obtains $a\left(t_{P l}\right) \approx 10^{-5} \mathrm{~m}$, so that for the scale of Grand Unification $m=10^{15} \mathrm{GeV}$, one has $10^{75}$ fermion particles with the energy of the Planckian order.

\section{Gravitational Radiation in Collisions of Trans-Planckian Superheavy Particles}

In our paper [8], we obtained the following result for the gravitational radiation energy in the collision of two particles with the energy $E_{\mathrm{c} . m .}<E_{P l}$ in the centre of mass system:

$$
E=\frac{4}{\pi} \frac{E_{\text {c.m. }}^{3}}{E_{P l}^{2}} \ln \left(\frac{E_{\text {c.m. }}}{E_{P l}} \frac{M_{P l}}{\sqrt{m_{1} m_{2}}}\right)
$$

where $M_{P l}=\sqrt{\hbar c / G}=2.18 \times 10^{-8} \mathrm{~kg}$ is the Planck mass and $m_{1}$ and $m_{2}$ are the masses of colliding particles. Note that even for such light particles as electrons, one has $\ln \left(M_{P l} / m\right)<52$. Therefore, one can see from (39) that if $E_{\mathrm{c} . \mathrm{m} .} \ll E_{P l}$, then gravitational radiation is small:

$$
E_{\text {c.m. }} \ll E_{P l} \Rightarrow \frac{E}{E_{\text {c.m. }}} \ll 1 .
$$

However, for $E_{\text {c.m. }} \geq E_{P l}$, the result is different. One has:

$$
E \approx \frac{4}{\pi} E_{\text {c.m. }} \cdot \ln \left(\frac{E_{\text {c.m. }}}{E_{P l}} \frac{M_{P l}}{\sqrt{m_{1} m_{2}}}\right) .
$$

This means that the role of gravitational radiation becomes large, and it can play the role of bremsstrahlung in electromagnetic radiation. In [8], it was shown that if colliding particles have nonzero electric charges, then the bremsstrahlung due to electromagnetism is much smaller than the gravitational one. Let us evaluate the order of the energy of this gravitational radiation.

Evaluate the gravitational background radiation of these particles supposing that the energy of gravitation is radiated in collisions at Compton epoch $t \approx \hbar / m c^{2}$ of the expanding Universe. Taking into account the decrease of the energy of gravitational waves due to the expansion of the Universe, one obtains in the modern epoch $t_{\text {mod }} \approx 10^{18} \mathrm{~s}$ the value of the energy as $10^{67} E_{P l} \sqrt{t_{C} / t_{\text {mod }}} \approx$ $10^{48} \mathrm{~J}$ for scalar particles and $\approx 10^{56} \mathrm{~J}$ for fermion particles. Therefore, the density of this energy of radiation $\approx 10^{-30} \mathrm{~J} / \mathrm{m}^{3}$ for the case of scalar particles or $\approx 10^{-22} \mathrm{~J} / \mathrm{m}^{3}$ for the case of fermion particles is much less than the energy of the electromagnetic background radiation equal to $\approx 4 \times 10^{-14} \mathrm{~J} / \mathrm{m}^{3}$.

Author Contributions: A.A.G. and Y.V.P. have contributed equally to all parts of this work. All authors read and agreed to the published version of the manuscript.

Funding: This research is supported by the Russian Foundation for Basic research (Grant No. 18-02-00461 a). The work of Y.V.P. was supported by the Russian Government Program of Competitive Growth of Kazan Federal University.

Conflicts of Interest: The authors declare no conflict of interest. 


\section{References}

1. Grib, A.A.; Mamayev, S.G. On field theory in Friedmann space. Yad. Fiz. 1969, 10, 1276-1281. [Engl. transl. in Sov. J. Nucl. Phys. 1970, 10, 722-725].

2. Grib, A.A.; Mamayev, S.G.; Mostepanenko, V.M. Vacuum Quantum Effects in Strong Fields; Friedmann Lab. Publ.: St. Petersburg, Russia, 1994.

3. Grib, A.A.; Pavlov, Y.V. Superheavy particles in Friedmann cosmology and the dark matter problem. Int. J. Mod. Phys. D 2002, 11, 433-436. [CrossRef]

4. Dubrovich, V.K.; Khlopov, M.Y. Primordial pairing and binding of superheavy charged particles in the early Universe. JETP Lett. 2003, 77, 335-338. [CrossRef]

5. Dubrovich, V.K.; Fargion, D.; Khlopov, M.Y. Primordial bound systems of superheavy particles as the source of ultra-high energy cosmic rays. Astropart. Phys. 2004, 22, 183-197. [CrossRef]

6. Grib, A.A.; Pavlov, Y.V. Do active galactic nuclei convert dark matter into visible particles? Mod. Phys. Lett. A 2008, 23, 1151-1159. [CrossRef]

7. Kolb, E.W.; Starobinsky, A.A.; Tkachev, I.I. Trans-Planckian wimpzillas. J. Cosmol. Astropart. Phys. 2007. [CrossRef]

8. Grib, A.A.; Pavlov, Y.V. On the limiting energy of the collision of elementary particles close to horizon of the rotating black hole. Mod. Phys. Lett. A 2020, 35, 2050262. [CrossRef]

9. Hooft, G. Graviton dominance in ultra-high-energy scattering. Phys. Lett. B 1987, 198, 61-63. [CrossRef]

10. Rubin, S.G.; Sakharov, A.S.; Khlopov, M.Y. The formation of primary galactic nuclei during phase transitions in the early Universe. J. Exp. Theor. Phys. 2001, 92, 921-929. [CrossRef]

11. Grib, A.A. Early Expanding Universe and Elementary Particles; Friedmann Lab. Publ.: St. Petersburg, Russia, 1995.

12. Birrell, N.D.; Davies, P.C.W. Quantum Fields in Curved Space; Cambridge University Press: Cambridge, UK, 1982.

13. Grishchuk, L.P.; Yudin, V.M. Conformal coupling of gravitational wave field to curvature. J. Math. Phys. 1980, 21, 1168-1175. [CrossRef]

14. Linde, A.D. Particle Physics and Inflationary Cosmology; Harwood Academic: New York, NY, USA, 1990.

Publisher's Note: MDPI stays neutral with regard to jurisdictional claims in published maps and institutional affiliations.

(c) 2020 by the authors. Licensee MDPI, Basel, Switzerland. This article is an open access article distributed under the terms and conditions of the Creative Commons Attribution (CC BY) license (http://creativecommons.org/licenses/by/4.0/). 